\title{
Successful growth of two different quantum dots on one substrate
}

\author{
C.F. Tsai ${ }^{a}$, Y.H. Changa, ${ }^{a}$, J.H. Cheng ${ }^{a}$, S.C. Yang ${ }^{a}$, C.C. Hsu ${ }^{a}$, Y.F. Chen ${ }^{a}$, L.C. Chen ${ }^{b}$ \\ ${ }^{a}$ Department of Physics, National Taiwan University, Taipei 106, Taiwan, ROC \\ ${ }^{\mathrm{b}}$ Center for Condensed Matter Sciences, National Taiwan University, Taipei 106, Taiwan, ROC
}

\begin{abstract}
We report the successful growth of ZnSe and ZnTe quantum dots (QDs) embedded in ZnS on GaAs substrate. These QDs have good optical properties and show quantum confinement effect. High-resolution electron scanning microscope studies show that these QDs are grown in Volmer-Weber mode. It is found that the size of the QDs is controlled by the growth duration. When the growth time is short, high density of QDs could be fabricated, but with a long growth time the small QDs get together to form a large cluster. We also show that with this growth method it is possible to grow both ZnSe quantum and $\mathrm{ZnTe}$ QDs on one substrate at the same time. For this dual QDs system, two peaks corresponding to the emission from the $\mathrm{ZnSe}$ dots $(3.0 \mathrm{eV}$, blue-violet) and $\mathrm{ZnTe}$ dots $(2.6 \mathrm{eV}$, green-blue) could be observed at the same time in the photoluminescence measurement.
\end{abstract}

(C) 2003 Elsevier B.V. All rights reserved.

PACS: 81.05.Dz; 81.05.Kk; 81.15.Gh

Keywords: Quantum dots; Light-emitting diodes; ZnSe; ZnTe

Quantum dots (QDs) systems have brought much attention because of their potential applications in the optical and opto-electronic devices [1-10]. Various ways of producing QDs have been demonstrated. Self-organized QDs attracted most attention because of their non-destructive nature. These QDs are found to grow under Stranski-Krastanov ( $\mathrm{S}-\mathrm{K})$ mode: a layer was first grown in two- and three-dimensional islands which was formed after some critical thickness was reached [11]. These dots were formed to relax strain energy but without creating dislocations between the dots and the underlying layer and are epitaxially and coherently grown. Recently, we showed

\footnotetext{
* Corresponding author. Tel./fax: 886-2-33635126.

E-mail address: yhchang@phys.ntu.edu.tw (Y.H. Chang).
}

that the high-quality QDs could be grown in a metal organic chemical deposition (MOCVD) system by controlling the flow duration of the precursors [4-6]. The basic idea is to embed QDs into another material with wider gap but without introducing a wetting layer. To do so a rough surface is first prepared as a starting layer. QDs were then grown on this surface, and the size of the QD is controlled by the growth duration. By using this method, we were able to grow good-quality ZnSe and ZnTe quantum dots embedded in $\mathrm{ZnS}$, and both QDs show substantial quantum confinement effect. In many applications, it is desirable to create light-emitting devices that could emit light with multi-colors such as a light-emitting diode that could emit white light. A possible way to make this kind of light-emitting devices is to grow dual QDs or tri-QDs 
system. In this paper, we report the successful growth of the dual QDs system by using the growth duration control method.

The samples were grown with a home-made MOCVD system. Dimethylzinc (DMZn), dielthylselenide (DESe), diethyltelleride (DETe), and diethylsulfide were used as precursor to grow $\mathrm{ZnSe}, \mathrm{ZnTe}$, and $\mathrm{ZnS}$, respectively. The $(100) \mathrm{GaAs}$ substrate was heated to $550^{\circ} \mathrm{C}$ for $15 \mathrm{~min}$ before the sample growth, a layer of $\mathrm{ZnS}$ of few hundred Angstrom was then grown on top of $\mathrm{ZnSe}$ at $530^{\circ} \mathrm{C}$ to create a rough surface. The growth temperature was then kept at $530^{\circ} \mathrm{C}$ and the growth pressure was kept at 760 Torr. At this growth temperature and pressure, the growth rate was calibrated to be 4,9 , and $7 \AA / \mathrm{s}$ for $\mathrm{ZnS}, \mathrm{ZnSe}$, and $\mathrm{ZnTe}$, respectively. For growing $\mathrm{ZnSe}$ QDs, after the $\mathrm{ZnS}$ growth, 10 periods of $40 \mathrm{~s} / 10 \mathrm{~s} / \mathrm{ns}$ (ZnS growth/growth interruption/ZnSe growth), growth sequence were carried out. Here the nanoseconds range is between 1 and $10 \mathrm{~s}$, and is the growth duration of the $\mathrm{ZnSe}$. After the growth, high-resolution field emission scanning electron microscope (SEM) was used to study the surface morphology of the samples. The photoluminescence (PL) properties of the samples were studied with a $\mathrm{He}-\mathrm{Cd}$ laser, a double monochromator, a lock-in amplifier and a $10 \mathrm{~K}$ closed cycle refrigerator.

The surface morphology of the $\mathrm{ZnS}$ and the effect of growth duration on the size of the QDs are displayed in Figs. 1(a)-(c). We could see in Fig. 1(a) that for $\mathrm{ZnS}$ grown on the GaAs substrate, the $\mathrm{ZnS}$ surface is made up of $\mathrm{ZnS}$ islands with an average size of a few tens of nanometers. From Fig. 1(b) we found that when the $\mathrm{ZnSe}$ growth duration time is $4 \mathrm{~s}$, ZnSe QDs with diameter of a few nanometer were grown on top of the $\mathrm{ZnS}$ islands. We could see in this figure that the dots are small but the density is high, QD density of around $5 \times 10^{11} \mathrm{~cm}^{-2}$ could be obtained with this growth condition. When the growth time increases, the size of the ZnSe QDs also increases. As could be seen in Fig. 1(c), when the growth duration time is $10 \mathrm{~s}$, large clusters show up and are the dominant feature of the surface. These high-resolution SEM pictures provide the evidence we claimed previously that it is not a $\mathrm{S}-\mathrm{K}$ growth, or a layer plus island growth, instead, the growth is a Volmer-Weber mode, or direct island growth [4-7]. An important feature to be noted from these pictures is that when the growth time
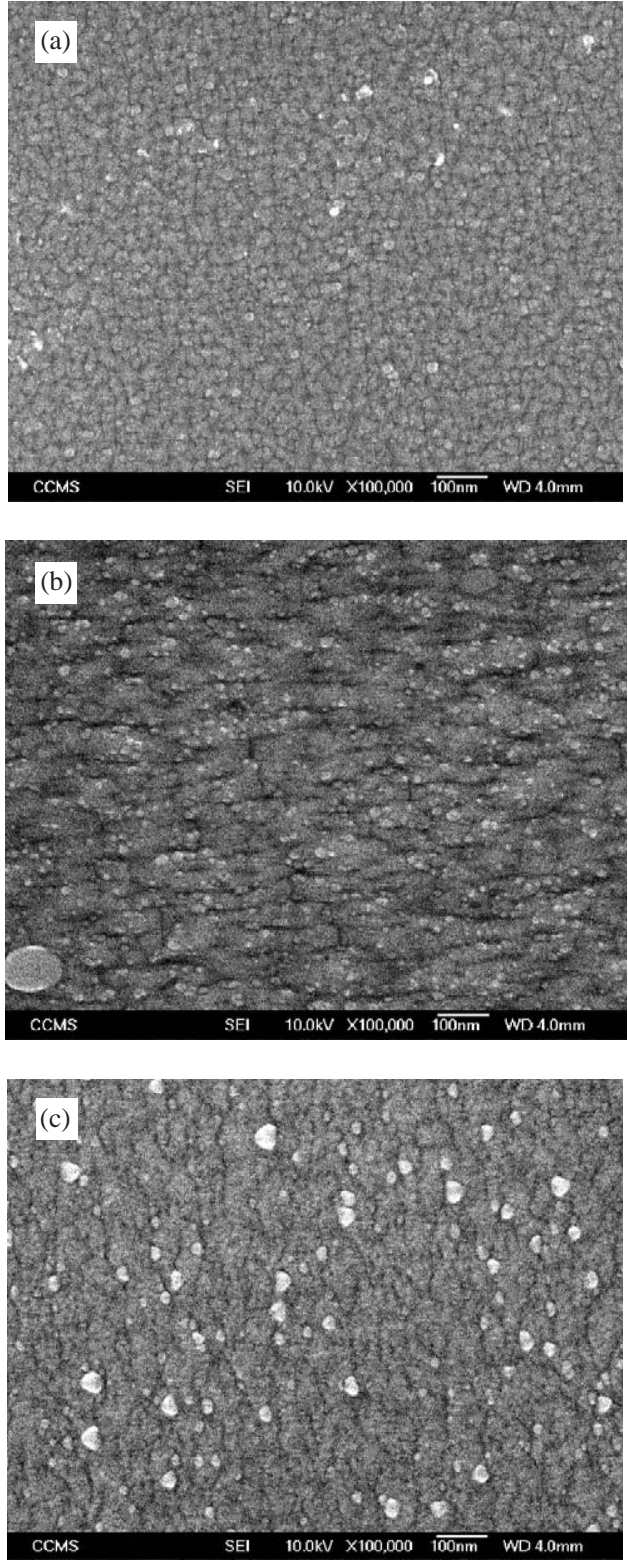

Fig. 1. (a) High-resolution SEM picture of the surface of $\mathrm{ZnS}$ layer grown on GaAs substrate. The surface consists of $\mathrm{ZnS}$ island with an average size of tens of nanometers, (b) with $\mathrm{ZnSe}$ growth time $4 \mathrm{~s}$ we could see many $\mathrm{ZnSe}$ QDs grown on top of $\mathrm{ZnS}$ islands, (c) with $\mathrm{ZnSe}$ growth time $10 \mathrm{~s}$, the $\mathrm{ZnSe}$ dots grows into cluster with size 10 times larger than the QDs in (b). The length of the white bars in these pictures is $100 \mathrm{~nm}$. 
is short, the QD size is also small. However, with long growth time, the density of the small dots did not increase. It appears that when the growth time is long, the small quantum dots merge together to form a big cluster, and the density of the smaller dots actually decreases. In the previous study we found that PL intensity decreases and sometimes disappears completely with increasing $\mathrm{ZnSe}$ growth time [4-6]. This is consistent with the SEM results which show that as the growth time increases, the density of small QDs that are responsible for the high efficient light emission decreases with growth time.

Unlike the S-K growth of QDs, our growth method does not rely on the mismatch of the lattice constant between the barrier and the QD materials. This unique property makes it possible to grow two different kinds of QDs in one substrate at the same time. In the growth of dual QDs system we grow $\mathrm{ZnSe}$ and ZnTe QDs embedded in ZnS alternatively for 10 periods in one growth round. The growth sequence is as follows: a few hundreds Angstrom of $\mathrm{ZnS}$ grown on GaAs substrate, $40 \mathrm{~s} / 10 \mathrm{~s} / \mathrm{ns}$ of $\mathrm{ZnS}$ growth/growth interruption/ZnSe growth, and $40 \mathrm{~s} / 10 \mathrm{~s} / \mathrm{ns}$ of $\mathrm{ZnS}$ growth/growth interruption/ZnTe growth. The first $40 \mathrm{~s} / 10 \mathrm{~s} / \mathrm{ns}$ sequence is for growing ZnSe QDs and the second $40 \mathrm{~s} / 10 \mathrm{~s} / \mathrm{ns}$ sequence is for growing $\mathrm{ZnTe}$ QDs. The two sequences were then repeated 10 times, so that we have a sample that contains 10 periods of alternating ZnSe and ZnTe QDs.

Temperature-dependent PL spectra of the dual QDs sample are depicted in Fig. 2. The growth duration of both QDs are the same, $2 \mathrm{~s}$. We could see that the spectra consist of two emission peaks. The peak with energy at around $2.63 \mathrm{eV}$ is attributed to the PL emission from the $\mathrm{ZnTe}$ QDs and the peak at around $3.06 \mathrm{eV}$ is attributed to the emission from the ZnSe QDs. These value are about $240 \mathrm{meV}$ larger than the PL emissions from bulk ZnTe $(2.39 \mathrm{eV})$ and $\mathrm{ZnSe}(2.82 \mathrm{eV})$ emission, respectively. These large blue shifts are due to the quantum confinement effect and are consistent with the previous experimental results. These results demonstrate that dual QDs were successfully grown by the growth duration method. It is worth noting that because the intensity of the emitted light depends on growth conditions, such as the growth duration, flow rate, etc, it is possible to vary the relative intensity of the two emission bands by changing the growth parameters of the MOCVD system.

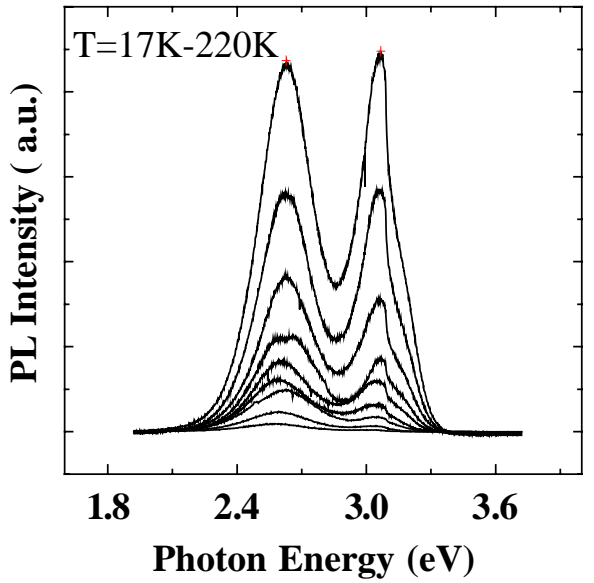

Fig. 2. PL spectra of the dual-QDs system taken, from top to bottom, $T=17,40,60,80,100,130,160,190$, and $220 \mathrm{~K}$. The low-energy peak is attributed to the PL emission from $\mathrm{ZnTe}$ QDs, and the high-energy peak is attributed to the ZnSe QDs.

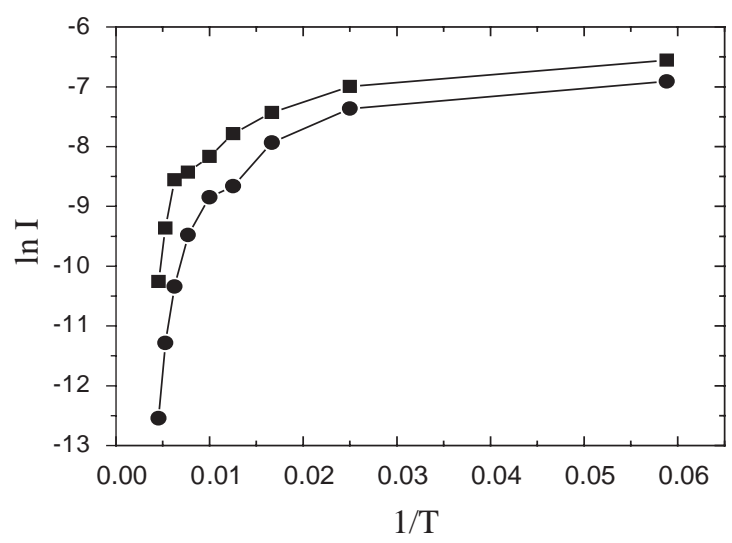

Fig. 3. Plot of $\ln I_{\mathrm{PL}}$ vs. $1 / T$ for $\mathrm{ZnTe}$ (upper curve) and $\mathrm{ZnSe}$ (lower curve).

The temperature dependence of the integrated PL intensity $\left(I_{P L}\right)$ is also studied. The quenching of the PL could be expressed as

$I_{\mathrm{PL}}(T)=\frac{I_{0}}{1+A_{1} \exp \left(-E_{\mathrm{A}} / k T\right)}$.

Here $k$ is Boltzman constant, $I_{0}$ is the intensity at the lowest attainable temperature and $E_{\mathrm{A}}$ is the activation energy responsible for the quenching of PL. By plotting $\ln I_{\mathrm{PL}}$ vs. $1 / T$, from the slope of the curve we could determine $E_{\mathrm{A}}$ (Fig. 3) and from these fittings 
we found that $E_{\mathrm{A}}=48.5 \mathrm{meV}$ for the ZnTe QDs and $E_{\mathrm{A}}=54.7 \mathrm{meV}$ for the ZnSe QDs. These numbers are close to the activation energy $(40 \mathrm{meV})$ obtained by Lu et al. [10] on MBE-grown ZnSe QD embedded in $\mathrm{ZnS}$. It was argued that the thermal quenching is either by the carrier escaping to the defect centers or the barriers. Since the conduction and valence band offset are a few hundred millielectronvolt for the $\mathrm{ZnS} / \mathrm{ZnSe}$ system, it is more likely that the quenching of PL is due to the escape of electron/hole to defect centers located at $\mathrm{ZnSe} / \mathrm{ZnS}$ or $\mathrm{ZnTe} / \mathrm{ZnS}$ interfaces.

In conclusion, the feasibility of using flow duration control to grow QDs with a MOCVD system was demonstrated. From the high-resolution SEM studies, we found that with this growth method the QDs were grown in Volmer-Weber mode. We also found that large amount of small QDs could only be obtained when the growth duration time is short. For the long-growth duration time, the QDs tend to merge together into large clusters. Dual QDs system with good optical properties was successfully grown. The sample could emit both the green and blue light simultaneously in the PL measurement. Quantum confined effect was observed in the dual-QD system, the quenching of the PL is studied and an activation of around $50 \mathrm{meV}$ is obtained.

\section{References}

[1] Y. Arakawa, H. Sakaki, Appl. Phys. Lett. 40 (1982) 939.

[2] M. Longo, N. Loverine, A.M. Macini, A. Passaseo, G. Leo, M. Mazzer, M. Berti, A.V. Drigo, Appl. Phys. Lett 70 (1997) 2256.

[3] Y.-h. Wu, K. Arai, T. Yao, Phys. Rev. B 53 (1996) R10485.

[4] M.C. Harris Liao, Y.H. Chang, Y.F. Chen, J.W. Hsu, J.M. Lin, W.C. Chou, Appl. Phys. Lett. 70 (1997) 2256.

[5] M.C. Harris Liao, Y.H. Chang, C.C. Tsai, M.H. Cheng, Y.F. Chen, J. Appl. Phys. 86 (1999) 4694.

[6] Y.H. Chang, M.H. Chieng, C.C. Tsai, M.C. Harris Liao, Y.F. Chen, J. Electron. Mater. 29 (2000) 173.

[7] C.A. Smith, H.W.H. Lee, V.J. Leppert, S.H. Rishbud, Appl. Phys. Lett. 75 (1999) 1688.

[8] T. Tawara, S. Tanaka, H. Kumano, I. Suemune, Appl. Phys. Lett. 75 (1999) 235.

[9] Z.H. Ma, W.D. Sun, I.K. Shou, G.K.L. Wong, Appl. Phys. Lett. 73 (1998) 1340.

[10] L.W. Lu, C.L. Yang, J. Wang, I.K. Sou, W.K. Ge, J. Appl. Phys. 93 (2003) 5325.

[11] D.J. Eaglesham, M. Serullo, Phys. Rev. Lett. 64 (1996) 1943. 